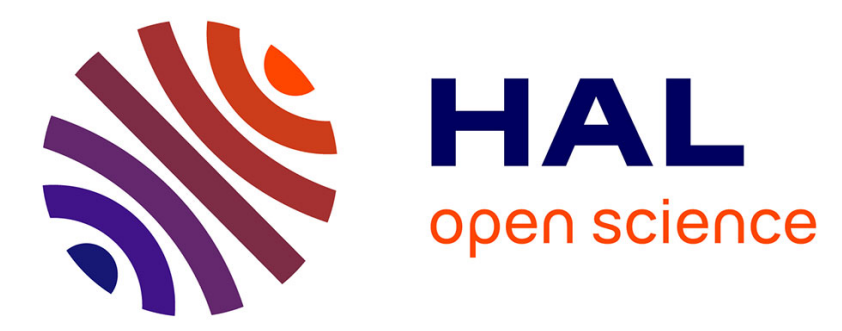

\title{
Culture politique réfractaire et décollage économique. L'exemple de la Vendée du Nord-Est
}

\author{
Jacques Palard, Thierry Berthet
}

\section{To cite this version:}

Jacques Palard, Thierry Berthet. Culture politique réfractaire et décollage économique. L'exemple de la Vendée du Nord-Est. Revue Française de Science Politique, 1997, 47 (1), pp.29-48. 10.3406/rfsp.1997.395147 . halshs-00105571

\section{HAL Id: halshs-00105571 \\ https://shs.hal.science/halshs-00105571}

Submitted on 23 Apr 2019

HAL is a multi-disciplinary open access archive for the deposit and dissemination of scientific research documents, whether they are published or not. The documents may come from teaching and research institutions in France or abroad, or from public or private research centers.
L'archive ouverte pluridisciplinaire HAL, est destinée au dépôt et à la diffusion de documents scientifiques de niveau recherche, publiés ou non, émanant des établissements d'enseignement et de recherche français ou étrangers, des laboratoires publics ou privés.

\section{(1)(1) $\$(0)$}

Distributed under a Creative Commons Attribution - NonCommercial - ShareAlikel 4.0 


\section{Culture politique réfractaire et décollage économique. L'exemple de} la Vendée du Nord-Est

Monsieur Jacques Palard, Monsieur Thierry Berthet

\section{Citer ce document / Cite this document :}

Palard Jacques, Berthet Thierry. Culture politique réfractaire et décollage économique. L'exemple de la Vendée du Nord-Est. In: Revue française de science politique, $47^{\mathrm{e}}$ année, $\mathrm{n}^{\circ} 1$, 1997. pp. 29-48;

doi : https://doi.org/10.3406/rfsp.1997.395147

https://www.persee.fr/doc/rfsp_0035-2950_1997_num_47_1_395147

Fichier pdf généré le 26/04/2018 


\title{
Résumé
}

Refractory political culture and economic takeoff in northeastern vendee

The bilateral links existing in a given social formation betlveen the organization of the political system and the economic fabric shed light on the process of local economic development. The article emphasizes the structuring of economic space by local socio-cultural elements, in a context of industrialization in a rural setting, according to the model of industrial districts. Northeastern Vendée provides an interesting example of a fragment in which local synergies facilitate the emergence of an industrial system characterized by the endo-geneity of its mode of development and the growth of a neftvork of small - and medium - sized companies. These local synergies range from the penetration of traditional agricultural organization to the vitality of a religious culture oriented toward the renewal of local elites.

\begin{abstract}
Refractory political culture and economic takeoff in northeastern vendee

The bilateral links existing in a given social formation betlveen the organization of the political system and the economic fabric shed light on the process of local economic development. The article emphasizes the structuring of economic space by local socio-cultural elements, in a context of industrialization in a rural setting, according to the model of industrial districts. Northeastern Vendée provides an interesting example of a fragment in which local synergies facilitate the emergence of an industrial system characterized by the endo-geneity of its mode of development and the growth of a neftvork of small - and medium - sized companies. These local synergies range from the penetration of traditional agricultural organization to the vitality of a religious culture oriented toward the renewal of local elites.
\end{abstract}




\title{
CULTURE POLITIQUE RÉFRACTAIRE ET DÉCOLLAGE ÉCONOMIQUE L'EXEMPLE DU NORD-EST VENDÉEN
}

\author{
THIERRY BERTHET, JACQUES PALARD
}

L es travaux des théoriciens, principalement anglo-saxons, de l'«économie politique» ont permis de mieux appréhender les rapports de réciprocité qui s'engagent au sein d'une formation sociale entre le mode de structuration du système économique et l'organisation du système politique ". Ils éclairent les processus du développement économique local, processus fortement différenciés selon le temps et le lieu dans la mesure même où ils constituent des «productions» spécifiques de configurations socio-politiques originales. Ce qu'il est convenu d'appeler l'«industrialisation diffuse » à caractère endogène, qui prend la forme de «districts industriels locaux », constitue une claire illustration de ce processus multifactoriel, dont ne saurait rendre compte la seule approche économique. Caractérisé par l'implantation de petites et de moyennes entreprises, notamment en milieu rural, et par une stratégie de spécialisation flexible ${ }^{2}$, ce modèle de développement est particulièrement bien implanté en Italie du Nord-Est (Vénétie et Trentin-Haut Adige) et du Centre (Émilie-Romagne, Ombrie, Toscane et Marches), couramment dénommée «troisième Italie ${ }^{3}{ }^{3}$. Il marque également de son empreinte des zones du territoire français, dont certaines comptent parmi les «régions qui gagnent» ${ }^{4}$. Le district du Choletais participe de ce type de dynamique originale, et singulièrement, en son sein, la Vendée du Nord-Est, qui retiendra ici l'attention. L'analyse s'attachera prioritairement à comprendre les effets des structures socio-spatiales et en particulier la prégnance du modèle culturel local, à forte composante religieuse, sur les conditions du développement du secteur industriel qui s'y est opéré à compter du début des années 1950.

1. Cf. E.S. Phelps, Économie politique, Paris, Fayard, 1990 (trad. franç.); L.C. Robbins, Politics and Economics: Papers in Political Economy, Londres, MacMillan, 1963.

2. Cf. M. Piore, C. Sabel, Les chemins de la prospérité, Paris, Hachette, 1989.

3. E. Ritaine, «La modernité localisée? Leçons italiennes sur le développement régional », Revue française de science politique, 39 (2), 1989, p. 154-177.

4. B. Ganne, «Place et évolution des systèmes industriels locaux. Économie politique d'une transformation», dans G. Benko, A. Lipietz (dir.), Les régions qui gagnent. Districts et réseaux: les nouveaux paradigmes de la géographie économique, Paris, PUF, 1992. 


\section{ISOLAT CHOLETAIS ET ENDOGÉNÉITÉ DU DÉVELOPPEMENT}

Le territoire du district industriel choletais se situe sur une partie de quatre départements: la Vendée, le Maine-et-Loire, les Deux-Sèvres et la Loire-Atlantique. Sa partie vendéenne couvre cinq cantons, qui appartiennent à la partie bocagère de la Vendée «militaire»: Les Herbiers, Montaigu, Mortagne-sur-Sèvre, Pouzauges et Saint-Fulgent.

Géographes, historiens, économistes et sociologues ont produit sur cette région des analyses dont la plupart s'inscrivent dans une démarche délibérément pluridisciplinaire. Dans le cadre de l'Action thématique programmée engagée par le CNRS à compter de la seconde moitié des années 1970 , une équipe de recherche nantaise conduite par Jean Renard a ainsi centré ses investigations sur les transformations économiques et sociales du canton de Saint-Fulgent. Plus récemment, des chercheurs grenoblois, membres de l'équipe IREP-Développement, ont présenté une analyse économique du phénomène de l'industrialisation diffuse dans la région du Choletais ${ }^{1}$ qui s'inspire du concept de «construction sociale du marché» développé par A. Bagnasco et C. Trigilia ${ }^{2}$ dans leurs travaux sur la «troisième Italie». Prenant appui sur ces études françaises et italiennes, l'approche politologique permet de prolonger l'analyse des facteurs socio-culturels du développement économique nord-vendéen. Elle s'est appuyée pour ce faire, outre l'investigation documentaire, sur des entrevues approfondies menées en septembre et octobre 1993 avec de dirigeants d'entreprises et de responsables politiques locaux.

\section{UN PROCESSUS LOCAL DE DÉVELOPPEMENT INDUSTRIEL}

«La tradition c'est le pied-mère, le progrès c'est le greffon». Cette expression, maintes fois entendue au cours de la recherche, rend parfaitement compte de ce qui, d'emblée, paraît effectivement relever d'une association des contraires: archaïsme et innovation, attachement au passé et dynamisme économique.

\section{Un fragment ante-républicain}

Pareille conjonction a suscité, parfois il est vrai dans des contextes singulièrement différents, des essais d'interprétation. L'analyse en termes de «fragmentation» que propose Louis Hartz dans The founding of new societies mérite à ce titre quelque attention: elle permet en effet d'éclairer, fût-ce sur le mode analogique, certaines des particularités socio-historiques du Nord-Est vendéen. Son étude historique de la construction des colonies de peuplement européennes d'Ancien Régime le conduit à construire un

1. G. Colletis, B. Pecqueur, C. Courlet, F. Winterhalter, Les systèmes industriels localisés en Europe, Grenoble, IREP-Développement, 1990, Série Rapports de recherche.

2. A. Bagnasco, C. Trigilia, La construction sociale du marché. Le défi de la troisième Italie, Cachan, Éditions de l'ENS-Cachan, 1993 (traduit par Thierry Berthet et Claudine Marenco). 


\section{Culture politique réfractaire et décollage économique}

modèle qui $s^{*}$ articule autour du concept de «fragment», transplant colonial de la métropole fortement marqué par une forme d'immobilisme social: «Lorsqu'un fragment d'une nation européenne est détaché de son système sociétal et projeté au loin sur un nouveau sol, il perd la dynamique de changement qui animait ce système et s'enfonce dans une sorte d'immobilité »'. Morceau d'Europe que cristallise la transplantation, le fragment colonial tend dès lors à accentuer ses traits identitaires et culturels. Mais. ainsi tenu à l'écart des mutations de sa formation sociale d'origine, il lui faut trouver en son sein les ressorts de son propre développement et de sa propre dynamique sociale. Selon L. Hartz, les fragments qui reposent sur les principes d'une société féodale font alors montre d'une savante alchimie entre tradition et novation: s'ils sont les plus lents à engendrer un processus de développement, ils sont aussi les plus créatifs au moment d'opérer le passage à la société industrielle.

Lorsque la perspective se déplace du contexte colonial à celui du territoire intra-métropolitain. le modèle interprétatif de $\mathrm{L}$. Hartz conserve quelque utilité. Mutatis mutandis, tout processus qui a pour effet de provoquer une mise en marge par rapport à la dynamique nationale dominante est en effet intrinsèquement porteur d'une forme d'«exil intérieur». Or, le NordEst vendéen ne présente pas seulement les traits d'un sous-ensemble territorial spécifique morphologiquement ancré dans une contrée de marches aux confins de la Bretagne, de l'Anjou et du Poitou. A l'écart des grandes voies de communication orientées vers la porte océane nantaise, le carrefour angevin et le seuil poitevin, il constitue aussi et surtout un univers clos, un isolat social dont la rupture révolutionnaire et le découpage politicoadministratif de la fin du $18^{\mathrm{e}}$ siècle sont venus encore renforcer une propension au repliement sur des formes micro-sociétales ${ }^{2}$. En observateur averti, André Siegfried estime, dans son Tableau politique de la France de l'Ouest sous la Troisième République ${ }^{3}$, que «la grâce déjà méridionale du paysage n'atténue pas, dans cette Vendée, je ne sais quoi d'isolé, de fermé. de réfractaire.». Ce relatif enclavement spatial et social paraît bien jouer ici, à titre de substitut fonctionnel. la fonction attribuée à la transplantation et à l'éloignement par l'analyse de Louis Hartz.

A l'enclavement géographique, s'ajoute ainsi la réclusion sur soi d'un groupe social marqué par l'histoire mouvementée et dramatisée de son rapport au «centre». La mémoire des «guerres de Vendée» - épisode à bien des égards fondateur. maintes fois rappelé, célébré et reconstruit - a joué ici un rôle crucial, qui a contribué à désigner cette région, selon les termes d'A. Siegfried, comme «un morceau oublié de la France du XIX ${ }^{e}$ siècle». une "grande région d'ancien régime». La mobilisation anti-révolutionnaire s'est nourrie par ailleurs, ainsi que le note le géographe Jean Renard ${ }^{4}$, d'un

1. L. Hartz, The Founding of New Societies, New York, Harcourt, Brace and World, 1964, p. 3 (traduction des auteurs).

2. Pour une analyse plus détaillée, on se reportera à l'ouvrage d'Alain Chauvet, Porte nantaise et isolat choletais, Maulévrier, Éditions Héraut, 1987, dont le chapitre 3 décrit les traits marquants du Bocage vendéen et. plus globalement, du «district» choletais.

3. Paris, Armand Colin, 1964 (1 ère éd. 1914).

4. J. Renard, Géopolitiqu' 'l's Pays de Loire, Saint-Sébastien, Éditions ACL. 1988. p. 62 et suiv. 


\section{Thierry Berthet, Jacques Palard}

antagonisme à l'égard des villes et de la bourgeoisie. Clairement inscrite dans le clivage ville-campagne, la révolte de 1793 et ses prolongements dans les décennies suivantes - jusqu'à la loi de séparation des Églises et de l'État et aux formes rémanentes de la question scolaire - révèlent et renforcent une idéologie à forte connotation ruraliste. Ainsi qu'on le verra plus avant, la religion, catholique en loccurrence, joue comme un facteur de légitimation à fonction identificatrice.

Bien qu'en position de dépendance à l'égard du pouvoir central. auquel elle reste institutionnellement subordonnée, la société-fragment, qu'elle soit coloniale ou infra-nationale. ne se pose en définitive qu'en s'opposant. Son développement ne saurait dès lors légitimement découler que de la mobilisation de ses propres ressources.

\section{Un développement endogène}

C'est un trait caractéristique de l'industrialisation du Nord-Vendée: la dynamique industrielle et la structuration du tissu économique est d'abord le fait non de la délocalisation d'entreprises extérieures mais d'entrepreneurs locaux'. Cette observation vaut d'ailleurs pour l'ensemble de l'arrondissement de Cholet, qui constitue la matrice du développement à l'œuvre dans le bocage vendéen: sur les 46 implantations d'entreprises qui y sont réalisées de 1960 à 1970, 39, représentant les quatre cinquièmes des 3350 emplois créés, relèvent d'initiatives locales ${ }^{2}$. Si le Sud du département de la Vendée bénéficie de l'arrivée d'entreprises extérieures, d'ailleurs concentrées dans les deux principales villes. La Roche-sur-Yon et Fontenay-le-Comte, le bocage constitue en revanche le terrain d'élection des entrepreneurs du cru.

Endogène par l'origine de ses initiateurs, le développement économique du bocage vendéen l'est également par son mode de financement. Lors de la première phase d'industrialisation du Nord-Est vendéen, au lendemain de la seconde guerre mondiale, la mobilisation des capitaux repose avant tout, sinon exclusivement, sur la contribution des réseaux familiaux ${ }^{3}$. Les institutions financières ainsi que les pouvoirs publics locaux et nationaux ne sont intervenus que dans un second temps, au terme d'une période d' «essai » ou de «mise à l'épreuve», selon l'expression d'un chef d'entreprise, ne contribuant ainsi qu'à la consolidation du processus engagé.

L'émergence de «capitaines d'industrie» suppose elle-même que s'engage un processus d'individuation — éminemment singulier dans un tissu social de type «communauté» où. de surcroît, les positions d'autorité sont héritées et socialement prescrites - et que se matérialisent socialement la propension et l'aptitude à prendre des risques économiques.

1. A. Chauvet, J. Renard. La Vendée. Le pays et les hommes, La Roche-sur-Yon. Ed. Le Cercle d'Or, 1978, p. 93-98.

2. G. Minguet, Naissance de l'Anjou industriel, Paris, L'Harmattan, 1985, p. 158.

3. B. Pecqueur, "Analyse de réseau et développement économique local: l'exemple du Choletais », Cahiers du LERASS. 20, mai 1990, p. 53. 
A quoi tient, à compter du début des années 1950, le foisonnement d'initiatives entrepreneuriales, nées dans le cadre de simples ateliers artisanaux et dont on peut observer que la plupart ont été couronnées de succès, aboutissant à la constitution de groupes industriels dont certains sont forts aujourd'hui de plusieurs centaines de salariés et dépassent parfois même le millier? La conduite d'entretiens auprès de chefs d'entreprise ainsi que l'analyse de biographies permettent de comprendre le contexte social dans lequel s'est inscrite la décision d'entreprendre prise par les pionniers du «modèle économique vendéen »'.

Il convient de noter, dès l'abord, l'origine des futurs entrepreneurs, issus dans une forte proportion de familles porteuses d'une tradition manufacturière ou artisanale (en particulier dans le domaine de la chaussure, de la confection et de la transformation du bois); cette observation rejoint celle des auteurs des travaux, déjà mentionnés, sur la «troisième Italie», qui soulignent la sous-représentation du milieu agricole dans le groupe des nouveaux chefs d'entreprises.

Sur le plan de la formation professionnelle, deux générations se sont succédé. La première a bâti sa réussite sur une pratique professionnelle associant étroitement savoir-faire artisanal — la maîtrise d'un métier - et introduction d'innovations techniques. La seconde génération a bénéficié d'une véritable formation, acquise le plus souvent au collège Saint-Gabriel, institution scolaire catholique située à Saint-Laurent-sur-Sèvre, aux confins de la Vendée et du Maine-et-Loire. Grâce à des choix pédagogiques novateurs, qui seront examinés plus avant, le collège Saint-Gabriel a permis de dispenser des formations professionnalisantes délibérément en phase avec les exigences du milieu industriel local. Il a aussi et surtout contribué à créer les conditions d'implantation de nouveaux secteurs d'activité : au tryptique traditionnel habillement-chaussure-bois sont venus s'adjoindre la construction des bateaux de plaisance et la construction métallique, les industries agroalimentaire et mécanique, les transports...

Les chefs d'entreprise nord-vendéens font montre, enfin, d'une forte propension à s'inscrire dans des réseaux professionnels, qui constituent une ressource stratégique de premier plan pour le développement des activités industrielles ${ }^{2}$. Traduction de formes de socialité marquées par un fort sentiment d'appartenance territoriale, ces réseaux remplissent de multiples fonctions, de l'échange d'informations sur les innovations techniques ou l'état du marché à la mise en commun de moyens et à la constitution de centres de ressources (formation professionnelle, agent multicarte à l'étranger...). Ils ont conduit par là à définir une forme de «concurrence disciplinée » ${ }^{3}$, qu'on observe à nouveau parmi les entrepreneurs de l'Italie du Centre et du NordEst et qui contribue à la formation et à l'intégration interne d'un véritable milieu industriel local.

1. L.M. Barbarit, L.M. Clenet, La nouvelle Vendée. Voyage dans la Vendée industrielle, Paris, Éditions France-Empire, 1990.

2. C. Courlet, B. Pecqueur, «Un modèle de développement diffus en mutation. L'exemple du Choletais ». Cahiers de l'IREP-Développement, 29, 1989, p. 14.

3. Ibid, p. 14; voir également G. Minguet, Chefs d'entreprise dans l'Ouest, Paris, PUF, 1993, qui présente une analyse des différentes instances d'expression des intérêts des chefs d'entreprises dans le Choletais et l'Anjou. 
Les études consacrées à la Vendée industrielle ont volontiers mis l'accent sur les facteurs socio-culturels du développement de l'entreprenariat et sur les formes prises par l'industrialisation diffuse. Celles qui ont porté sur ces mêmes processus dans la «troisième Italie» ont en outre porté leur attention sur le marché local du travail. Ce marché est évidemment marqué par le milieu rural ambiant, où la famille élargie, jusqu'à une date récente, a exercé des fonctions centrales: socialisation des jeunes à différentes formes de travail manuel et à l'exercice de responsabilités dans une économie d'auto-subsistance, inculcation des modèles d'autorité et des principes de solidarité communautaire...'. On en retrouve les traits dans le Nord-Est vendéen, où s'observe aussi la même disponibilité d'une main-d'œuvre jeune, dont l'importance numérique tient à la vitalité démographique et qui outrepasse les besoins des seules exploitations agricoles.

Le mode de faire-valoir de ces exploitations constitue l'une des composantes déterminantes des structures sociales locales. C'est en parcourant une carte du recensement de 1901 qu'A. Bagnasco constate entre les zones d'implantation du métayage et du fermage à cette époque et celles du développement contemporain de l'industrialisation diffuse une nette correspondance dont l'interprétation lui paraît justiciable d'une analyse en termes d'homologie structurale. A la différence de l'agriculture de type capitalistique, métayage et fermage reposent sur une autonomie relative du producteur individuel: les exploitations familiales représentent de véritables unités de production dotées d'une «structure sociale organisée, avec une division interne du travail, des rôles différenciés, des structures hiérarchiques ainsi que des processus internes de socialisation et de contrôle appropriés ${ }^{2}$. Mais le fermage et, plus encore, le métayage reposent aussi sur un contrôle externe de l'exploitation. La distribution des rôles internes et externes à l'unité de production induit de la sorte des comportements qui combinent autonomie et hétéronomie, indépendance et dépendance et qui traduisent les représentations localement dominantes de l'ordre social.

S'agissant de la Vendée, l'étude des modes de faire-valoir et de leurs effets socio-politiques n'opère pas en terrain vierge. Les analyses de la Vendée bocagère, à commencer par le Tableau politique de la France de l'Ouest d'André Siegfried, ont mis en évidence le poids de la grande propriété foncière, dont l'exploitation, jusqu'aux années 1960, a précisément reposé dans une large mesure sur le métayage.

Régis en majeure partie par le statut du métayage et du fermage, les exploitants du Bocage sont insérés dans un tissu de liens socio-économiques apte à préparer à l'intégration au milieu industriel une main-d'œuvre formée à la fois à l'autonomie que procure la gestion quasi indépendante du travail agricole, et à la sujétion au propriétaire foncier, véritable «maître» des lieux.

1. A. Bagnasco, C. Trigilia, La construction sociale du marché, op. cit., p. 64 et suiv.

2. Ibid., p. 115 . 
DÉPARTEMENT DE LA VENDÉE

Répartition des modes de faire-valoir des exploitations agricoles par région en 1929

(En pourcentage du nombre d'exploitants)

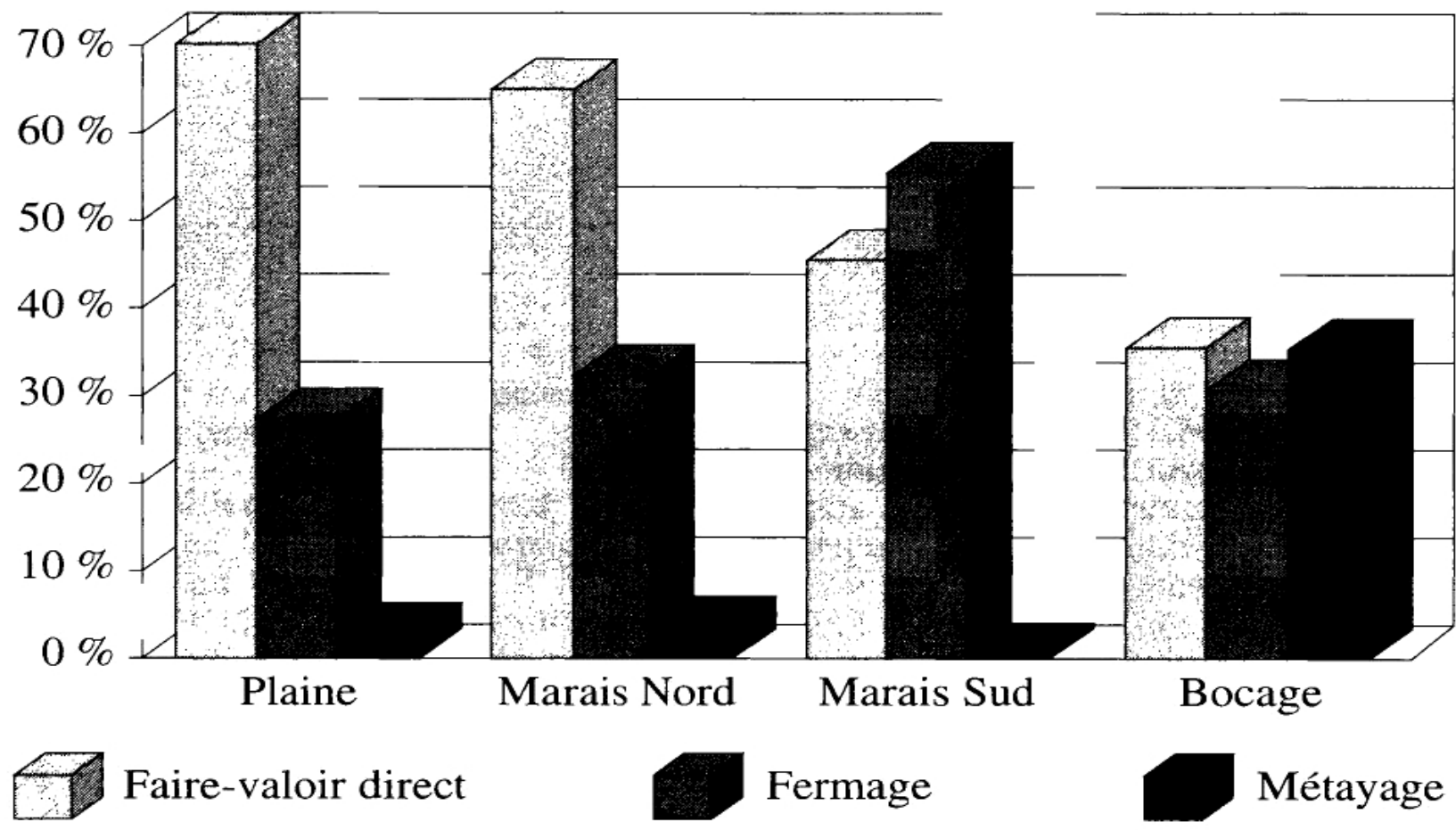

Source: Ministère de l'Agriculture, Monographie agricole du département de la Vendée, enquête de 1929. La répartition territoriale des modes de faire-valoir en vigueur en Vendée apparaît clairement différenciée. Presque absents des autres secteurs du département, les métayers représentent au contraire, dans le Bocage, plus du tiers des exploitants agricoles.

Les chefs d'entreprise ont, en toute logique, une représentation très positive d'une main d'œuvre aussi appliquée. Lors des entretiens, ils évoquent volontiers sa capacité d'adaptation ainsi que ses qualités morales: le courage, l'amour du travail bien fait, la conscience professionnelle, l'attachement à l'entreprise et le souci de sa réussite, conditionnés toutefois par des formes de «reconnaissance» et de respect en provenance des «patrons». Ces qualités sont souvent rapportées à la sauvegarde d'un «certain esprit rural, basé sur l'esprit du travailleur qui fait bien son métier, qui est sérieux, qui est consciencieux, qui prend des initiatives et sur qui on peut compter sans problèmes» (dirigeant d'une entreprise de construction métallique, Les Herbiers); «(Les employés) ne sont pas rancuniers; ils sont très attachés, je crois, à leur terroir, à leur entreprise, à leur hiérarchie» (dirigeant d'une entreprise de transports routiers, Montaigu). Il se dégage de ces appréciations élogieuses une impression d'ensemble qui corrobore l'hypothèse de la relation autonomie/dépendance. Capables d'initiatives et attachés à leur entreprise comme à un patrimoine propre (ce qui a grandement compté dans la conception de formes de gestion nouvelles et consensuelles de ressources humaines), la majorité des salariés ne remet pas en cause les modes paternalistes d'organisation des entreprises. On ne doit toutefois pas considérer cette légitimation du leadership social exercé par les chefs d'entreprise, ni la faiblesse de l'implantation syndicale, ni la rareté des conflits sociaux 
comme la résultante d'une attitude de résignation; ce sont plutôt les effets d'un système d'échange social, fait de sollicitude et de sollicitation, qui accorde úne large place aux procédures d'innovation concertée (flexibilité du temps de travail, instauration d'un crédit d'heures, intéressement des salariés aux résultats de l'entreprise...) L'absence de solution de continuité, dans l'activité de nombre des ouvriers ruraux (très souvent propriétaires de leur logement '), entre l'usine et la ferme familiale ${ }^{2}$ a pu contribuer à introduire et à perpétuer dans le milieu industriel les acquis culturels du faire-valoir indirect en agriculture.

Bien que se «greffant» sur une économie locale à dominante agraire, l'industrialisation du Nord-Est vendéen s'est ainsi opérée sans bouleversement social profond. La continuité l'emporte sans conteste sur les ruptures; on en peut observer les effets jusque dans la recomposition de la hiérarchie sociale et de la classe politique locale, qui opère ici également par homologie structurale: hier dominée par des hobereaux détenteurs de la propriété foncière, la vie politique municipale et cantonale l'est aujourd'hui par des entrepreneurs industriels ou des cadres supérieurs des entreprises, signe indiscutable de la légitimité dont celles-ci ont su bénéficier au sein de la société locale. Parce qu'elle ne s'est pas construite contre la société traditionnelle, l'usine a été socialement bien acceptée. Parce qu'il n'a pas entraîné une forte urbanisation -_ jugée néfaste par l'Église, qui y voit traditionnellement un vecteur de déstructuration sociale - le développement industriel de la Vendée choletaise a donné au système traditionnel de valeurs et de normes, qui risquait autrement d'être frappé d'obsolescence, une nouvelle assise sociale et de nouveaux espaces d'action. «Dans ce contexte, c'est en tenant ferme sur ses bases que la communauté territoriale assume sa rencontre avec 1'univers industriel: ce sont les normes et valeurs de la culture traditionnelle qui vont constituer la matrice de la culture mise en auvre pour intégrer l'usine dans le système social local; le travail à l'usine, les rapports de production eux-mêmes vont se trouver investis par les représentations traditionnelles $\gg^{3}$.

\section{ENTREPRENARIAT NORD VENDÉEN ET CULTURE CATHOLIQUE}

D'emblée, bien que de façon implicite, nous avons accordé à l'empreinte religieuse du «fragment» vendéen un rôle important dans la constitution des conditions et des formes du processus d'industrialisation qui s'y est développé. Adoptant une démarche tout à la fois inductive et récursive, c'est sur ce point qu'il convient désormais de revenir pour l'expliciter

1. N. Croix, «Les ouvriers du canton de Saint-Fulgent», Cahiers nantais, 19, janvier 1981 .

2. L. Rondeau, «Les ouvriers d'usine en milieu rural. Mortagne-sur-Sèvre en Vendée», thèse de doctorat en psychologie sociale, Université de Nanterre, 1970.

3. N. Mercier, D. Segrestin, «Des ouvriers sur leurs terres: deux études de cas comparées», Sociologie du travail, 2, 1983, p. 151. 
et l'approfondir, afin de mieux appréhender les fondements culturels des formes prises par l'innovation économique.

La question de la délimitation territoriale est ici centrale: le géographe Alain Chauvet observe à cet égard qu'il est

«étrange de constater la permanence de cet isolat à travers le temps: la nébuleuse industrielle en milieu rural, qu'on peut appeler "Vendée choletaise", correspond au foyer principal de l'insurrection de 1793. La Vendée contre-révolutionnaire était déjà auparavant un pôle de résistance à la réforme venue de La Rochelle, comme à la Renaissance arrivée par la Loire. Enfin, quelques siècles plus tôt, elle formait un bastion convoité par les Aquitains, les Francs et les Bretons ... Les moyens de communication actuels ont ouvert cette enclave, mais l'intégration entre l'homme et ses lieux ne peut se rompre en un jour; entre le lieu et l'utopie, entre l'isolat géographique choletais et l'utopie vendéenne, le lien n'est peut-être pas rompu »'.

Jean Renard constate, pour sa part, non seulement, à titre de «donnée permanente », un clair dimorphisme entre le bocage et la plaine mais aussi une différenciation au sein même du bocage: «Il y a un bloc des cantons de Nord-Est qui se soude au Choletais voisin pour constituer le cœur de la "Vendée catholique" ... Les corrélations avec d'autres facteurs: métayage, surcharge d'actifs agricoles, poids de la grande propriété, ne sont pas fortuits et confirment ces aspects socio-politiques ${ }^{2}$.

Toutefois, les interrogations du politologue ne trouvent qu'une réponse incomplète dans une méthode d'investigation qui établit un simple rapport de convenance ou une relation de corrélation là où l'analyse paraît ne pas manquer d'atouts pour avancer sur la voie de l'explication causale, dans la ligne, par exemple, du propos qu'inspire à Laurence Wylie l'étude des structures socio-culturelles de la commune de Chanzeaux, dans le Maine-etLoire voisin: "S'il fallait choisir un trait distinctif qui signale Chanzeaux parmi l'ensemble des villages de France, ce serait l'importance du rôle qu'y joue la religion catholique ... L'église est le point de ralliement des activités sociales de la commune ${ }^{3}$.

En cherchant à tisser les fils pour faire apparaître une trame sociale d'ensemble propre à rendre compte de la cohérence des comportements audelà des stratégies entrepreneuriales individuelles, l'analyse étologique soulève à l'évidence des difficultés d'ordre méthodologique, celles-là mêmes que pose à Max Weber, toutes proportions gardées, l'interprétation du rapport de congruence qui s'établit entre éthique protestante et esprit du capitalisme. Faut-il se contenter de relever une correspondance socio-spatiale entre l'une et l'autre? Est-on, au contraire, fondé à établir entre celle-là et celui-ci un lien de causalité, qui ferait de la première une sorte de matrice culturelle du second? Mais alors, quels indicateurs retenir, quels chaînons, notamment d'ordre institutionnel, privilégier pour expliquer cette filiation? Lorsque Jean Dumas s'interroge sur l'existence de «capitaines d'industrie» en Aquitaine et relève que «c'est l'absence d'entrepreneurs purs qui souli-

1. A. Chauvet, Porte nantaise et isolat choletais, op. cit., p. 171-172.

2. J. Renard, Géopolitique des Pays de Loire, op. cit, p. 181.

3. L. Wylie, Chanzeaux, village d'Anjou, Paris, Gallimard. 1970. 


\section{Thierry Berthet, Jacques Palard}

gne le mieux, en négatif, l'originalité régionale »', il se soumet au même type de démarche: dégager des régularités et en rendre compte sur la longue durée.

L'analyse du système de relations susceptibles de s'établir entre culture politique réfractaire et développement économique suscite deux questions majeures:

- peut-on rapporter l'«esprit du capitalisme vendéen» à l'«éthique catholique »?

- quelles sont les structures et les médiations institutionnelles qui permettent de rendre compte de cette éventuelle relation, d'en mesurer la portée et d'en dégager la signification?

\section{ÉTHIQUE CATHOLIQUE ET ESPRIT DU CAPITALISME}

Chacun connaît le constat que tire Max Weber de l'observation des acteurs économiques de l'Allemagne de son temps: «Les chefs d'entreprise et les détenteurs de capitaux, aussi bien que les représentants des couches supérieures qualifiées de la main-d'œuvre et, plus encore, le personnel technique et commercial hautement éduqué des entreprises modernes sont en grande majorité protestants. » Chacun sait aussi les termes de son interprétation: «Le principe de ces attitudes ... ne doit pas être recherché uniquement dans des circonstances extérieures temporaires historico-politiques, mais dans le caractère intrinsèque et permanent des croyances religieuses »2. Partant, en particulier, des admonitions morales d'un Benjamin Franklin, il fait observer que le désir d'accroître son capital est avant tout la traduction d'une éthique particulière «entièrement dépouillée de tout caractère eudémoniste, voire hédoniste »; le gain est devenu une fin en soi et le devoir s'accomplit dans l'exercice d'un métier. C'est là l'expression de l'ascétisme protestant, dont la dimension sotériologique conduit ses adeptes à conjuguer procès d'accumulation et refus des jouissances immanentes.

On ne saurait, sans d'extrêmes précautions, transposer ce cadre explicatif dans l'un des systèmes sociaux de la France de l'Ouest, qui lui est à première vue diamétralement opposé: milieu rural $v s$ milieu urbain, tradition catholique $v s$ tradition protestante, système de type «communauté » $v s$ système de type «société ». Il n'est toutefois pas illégitime de rechercher entre les deux modèles culturels non seulement des correspondances mais aussi des structures partiellement homologues et des substituts fonctionnels. La démarche, en l'occurrence, conduit à privilégier l'examen de parcours individuels. Le texte qu'écrit en 1980 l'épouse du fondateur de l'un des principaux groupes industriels locaux prend, de ce point de vue, valeur exemplaire. Il se présente sous la forme d'une lettre adressée au dernier fils de la famille, alors âgé de 18 ans, qui a lancé quelques jours plus tôt le

1. J. Dumas, «Y a-t-il eu des capitaines d'industrie en Aquitaine?», dans Hubert Bonin (dir.), Cinquante ans d'Aquitaine (1945-1995), Bordeaux, L'Horizon chimérique, 1995. L'auteur poursuit: «Les initiatives manufacturières sont constamment dans la dépendance de donneurs d'ordres extérieurs qui fixent les conditions du débouché et accaparent les risques et les bénéfices de la création... A la différence de ce que les analystes des «districts industriels» dégagent pour la région de Prato en Italie, pour celle de Solingen en Allemagne ou même pour le Choletais en France, il n'y a jamais eu de "ligne Aquitaine" pour les produits issus de ses activités» (p. 135).

2. M. Weber, L'éthique protestante et l'esprit du capitalisme, Paris, Plon, 1964, p. 31-33 ( 1 ère éd. allemande de: 1905). 
reproche cinglant: «Vous, les parents, vous ne vivez que pour l'argent et pour le boulot: la famille ne compte pas.» Dans la longue réponse de neuf pages, à usage strictement familial et qui vaut sans doute pour son auteur testament moral, la réussite de l'entreprise est explicitement associée à une consommation domestique délibérément faible, elle-même commandée avant tout par l'obligation morale d'assurer aux salariés de l'entreprise la sécurité d'emploi. Dans un tel système de valeurs, où l'éthique ascétique est directement rapportée au sens de la responsabilité, nulle place ne saurait être accordée à la recherche du pur accomplissement individuel :

«Du plus loin que je me rappelle, j'ai toujours entendu parler économie... «ménager» la chandelle, «ménager» les vêtements, qui devaient servir plusieurs années ... Nous avons toujours considéré que nous avions un engagement moral ${ }^{1}$ envers les ouvriers qui nous faisaient confiance, et qui nous donnaient leur travail et leur temps: il y avait entre nous un contrat ... Nous avons vécu la grande aventure d'entreprendre, et de réussir. Nous avons travaillé beaucoup, surtout au début où nous étions seuls pour faire face à tous les problèmes techniques, financiers, commerciaux, comptables, etc. Je ne regrette rien car nous n'avons jamais travaillé personnellement, mais avec et pour ceux qui étaient avec nous. Nous permettons à 250 familles de gagner leur vie. Notre vie a été donnée à $100 \%$. Pas pour la production à tout prix comme on t'apprend à nous le reprocher, mais pour répondre à l'engagement moral dont je te parlais plus haut. Et je souhaiterais que nos enfants vivent eux aussi cet idéal car, s'il en était autrement, très rapidement ils cesseront de s'entendre et l'entreprise périclitera."

Il n'y a dans cet argumentaire à visée auto-justificatrice nulle référence explicite au fondement religieux de l'engagement professionnel et de la réussite économique. Il n'en est pas moins clair qu'on ne peut l'analyser sans le rapporter à ses conditions sociales et culturelles d'élaboration et, par là, à la dimension religieuse de ces conditions, si du moins l'on s'accorde à donner ici de la religion une définition non pas substantive mais fonctionnelle; si, également, l'observateur s'engage dans la voie de l'élucidation à la fois des propriétés du contexte dans lequel émerge le processus d'industrialisation et du «détour»symbolique qui donne son sens et sa portée à l'action de la nouvelle classe d'entrepreneurs au sein de sa «communauté » d'appartenance.

\section{LES STRUCTURES DE LA MATRICE CATHOLIQUE}

Comme la double référence à $L$. Hartz et à la période contrerévolutionnaire l'a déjà signifié, la culture nord-vendéenne est avant tout une culture de résistance, une culture «réfractaire», au sens que prend ce terme lorsqu'il réfère aux prêtres qui ont refusé de prêter serment à la Constitution civile du clergé. De l'opposition exacerbée qui se développe au cours de la période révolutionnaire et que n'atténueront ni la paix concordataire ni la volonté d'apaisement du pouvoir napoléonien, naît une méfiance durable à l'encontre du pouvoir central; A. Chauvet y voit justement l'origine d'un surcroît d'énergie des communautés locales à assurer elles-mêmes leur survie

1. Souligné dans le texte. 


\section{Thierry Berthet, Jacques Palard}

et leur développement, au travers du maintien d'une forte fécondité et d'une capacité d'innovation de l'agriculture et de l'artisanat, puis de l'industrie'. On ne saurait toutefois se satisfaire d'une explication qui établirait une relation «immédiate» entre une représentation du passé et les formes présentes de vie collective. La force d'une telle représentation, fût-elle fondatrice, est conditionnée par la création ou le renforcement de formes institutionnelles d'intermédiation, qui en assurent tout à la fois la survivance et la légitimité.

C'est principalement dans le champ religieux que s'inscrivent ces formes institutionnelles, qu'il s'agisse de la paroisse, des établissements catholiques d'enseignement ou des mouvements d'Action catholique de jeunes, singulièrement de la Jeunesse agricole catholique (JAC) ${ }^{2}$. Il convient d'en faire le constat sans toutefois renoncer à y voir, à la suite de Max Weber, des productions sociales dont l'archéologie des formes d'émergence et d'imposition renvoie en dernière instance à la forte hiérarchisation des rapports sociaux. Emmanuel Todd note à cet égard que «le catholicisme ne définit ... pas seul la Contre-Révolution. L'amour du roi et des nobles doit, pour vivre, s'appuyer sur la métaphysique autoritaire et inégalitaire produite par certains types familiaux. La famille souche, autoritaire et inégalitaire, est le support anthropologique idéal de la Contre-Révolution. L'autorité du père nourrit celle du roi et du noble. L'inégalité des frères rend l'acceptation des inégalités sociales naturelle. Globalement, la famille souche définit un idéal invincible de hiérarchies ${ }^{3}$.

La place accordée aux structures religieuses dans notre démarche interprétative tient à leur prépondérance et aux traits de l'ordre social qui naît de leur processus d'institutionnalisation et de naturalisation. A. Siegfried souligne à ce propos l'étroitesse de la relation qui s'instaure entre la dépendance sociale et la dépendance spirituelle d'un «peuple à la fois passif et passionné », entre la forte hiérarchisation des rapports sociaux - «le régime politique du Bocage vendéen se définit d'un mot, c'est une hiérarchie»et le poids exercé par «l'autorité discrétionnaire du représentant de l'Église ${ }^{4}$. Ces structures religieuses pèsent inévitablement sur les conditions dans lesquelles se développe la formation d'un nouvel entreprenariat industriel. Il n'est pas interdit de penser que s'opère alors une forme de substitution fonctionnelle d'un type d'aristocratie à un autre, et que s'ouvre une voie par laquelle, conformément à l'une des vues prédictives de Tocqueville, «l'industrie pourrait bien à son tour ramener les hommes vers $l^{\prime}$ aristocratie ${ }^{5}$. Les effets indirects des transformations économiques sur

1. A. Chauvet, Porte nantaise et isolat choletais, op. cit., p. 65-66.

2. E. Ritaine, («La modernité localisée?», art. cité) rencontre, dans son analyse du district industriel de Prato, des formes d'emprise similaires de l'Église et des réseaux catholiques.

3. E. Todd, L'invention de l'Europe, Paris, Le Seuil, 1990, p. 210. C'est nous qui soulignons.

4. A. Siegfried, Tableau politique de la France de l'Ouest sous la Troisième République, op. cit., p. 25-26. Les analyses de L. Wylie, ainsi que celles du Groupe de sociologie rurale (CNRS) conduites sous la direction de $M$. Jollivet et d'H. Mendras (Les collectivités rurales françaises. Étude comparative de changement social, Paris, Armand Colin, 1971, t. 1), sont en cela parfaitement concordantes.

5. A. de Tocqueville, De la démocratie en Amérique, Paris, Gallimard, 1951, ( 1 ère éd.: 1835-1840), t. 1, chap. «Comment l'aristocratie pourrait naître de l'industrie», p. 164. 


\section{Culture politique réfractaire et décollage économique}

l'émergence d'un nouveau personnel politique et de nouvelles formes de la régulation sociale, au sein de l'entreprise comme au sein de la société locale, sont de nature à fonder pareille perspective.

\section{L'instance paroissiale}

Jusqu'aux années 1960, la paroisse constitue la véritable cellule de base non seulement de l'institution religieuse proprement dite mais bien de l'organisation sociale dans son ensemble, au point que commune et paroisse demeurent jusqu'à une date récente des termes équivalents et interchangeables. Les exemples abondent de ces unités territoriales qui développent un sentiment d'appartenance principalement alimenté par les nombreuses activités paroissiales. La société locale se pense et vit d'abord comme «société paroissiale »', sans doute différenciée socialement mais unie dans ses pratiques, à commencer par ses pratiques religieuses. On en a une claire traduction dans les nombreux ouvrages, mi-analyses, mi-témoignages, de chroniqueurs locaux ${ }^{2}$.

$S$ 'il fallait retenir du Bocage un indicateur majeur et durable de sa physionomie religieuse, c'est, classiquement, du côté de l'assistance à la messe dominicale qu'il conviendrait de le chercher ${ }^{3}$. L'enquête de pratique religieuse réalisée en 1957 dans le diocèse de Luçon ${ }^{4}$ (dont les limites sont celles du département de la Vendée) fait apparaître une profonde hétérogénéité territoriale des comportements religieux et met en évidence à nouveau la spécificité du Bocage.

L'assistance à la messe dominicale prend, dans le Bocage, un caractère quasi unanime. C'est dire qu'elle est aussi le fait des ouvriers, dont les enquêtes de pratique religieuse soulignent le plus souvent, ailleurs, la faible propension à fréquenter les lieux de culte: on compte en $195686 \%$ d'ouvriers «messalisants» dans les cantons de Saint-Fulgent et des Herbiers. Les calculs qui sont effectués par le clergé à l'occasion des visites canoniques de l'évêque de Luçon en 1964 montrent un début d' «effritement», qui ne remet cependant nullement en cause la netteté de cette différenciation territoriale du catholicisme vendéen.

C'est à travers l'attachement à la paroisse, c'est-à-dire à un espace spatialement restreint mais socialement actif, que se joue la possible résistance

1. J. Renard, Géopolitique des Pays de Loire, op. cit., p. 179.

2. Tel celui de H.-V. Mallard, La Verrie, bourg vendéen. Deux siècles d'histoire locale de la fin de l'Ancien Régime à nos jours, Maulévrier, Éditions Hérault, 1992. Évoquant la période de "la rupture de l'ordre ancien sous la Révolution française», l'auteur, lui-même maire de La Verrie et enseignant à la Faculté de droit d'Angers, insiste sur «la force du sentiment religieux»: «De la variété des groupes sociaux qui forment la communauté verraise, il serait inexact de conclure à la coexistence dans la même localité de trois ou quatre mondes imperméables. Au-delà de ce qui les sépare, il existe entre eux une parenté d'inspiration et une solidarité de fait nées d'une commune appartenance à un univers mental et religieux: la paroisse ... La paroisse est le cadre institutionnel dans lequel les uns et les autres expriment leurs convictions chrétiennes, se sentent étroitement pris en charge par un clergé conscient de l'importance de son rôle, prennent des décisions non seulement sur la gestion des biens d'Église mais encore sur l'administration des intérêts matériels de la Cité.»

3. Les recherches statistiques ont été réalisées sur ce point par Cécile Vrignon.

4. A l'instar de la plupart des autres diocèses français, généralement à l'instigation et sous la direction du chanoine Boulard. 
Taux de messalisants par canton en 1957

(En pourcentage de la population âgée de 7 ans et plus)

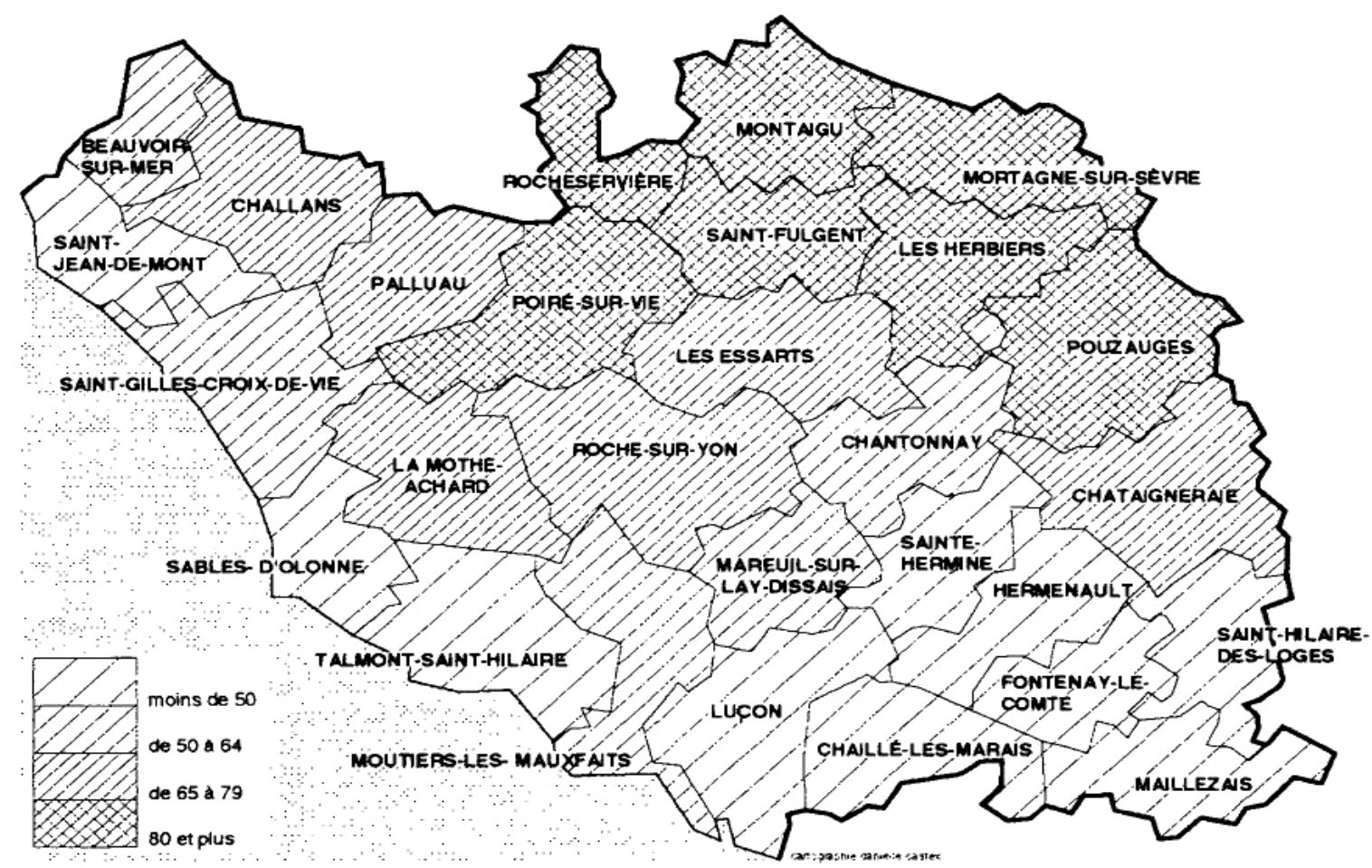

Source: Archives du diocèse de Luçon. Alors que dans les cantons du Sud et de l'Ouest côtier le taux de pratique dominicale est le plus souvent inférieur à $50 \%$ (Maillezais, $31 \%$; Luçon, $34 \%$; Fontenay-le-Comte, $36 \%$; Les Sables-d'Olonne, $47 \% \ldots$ ), avec un minimum de $21 \%$ à Chaillé-les-Marais, elle dépasse partout $80 \%$ dans le Nord-Est du département: La Rocheservière et Saint-Fulgent, $93 \%$; Les Herbiers, $91 \%$; Mortagne-sur-Sèvre, $88 \%$, Pouzauges, $85 \%$; Montaigu, $81 \%$.

à l'exode rural et à l'attractivité croissante de la ville. Outre la contribution financière des réseaux familiaux, la mobilisation des premiers capitaux provoquée par le mouvement d'industrialisation de l'après-guerre s'opère dans le cadre de caisses paroissiales - ancêtres des bureaux locaux du Crédit Mutuel -, dirigées par le clergé et ordonnées indirectement à la fixation de la main-d'œuvre disponible par la création de nouveaux emplois. Dans sa présentation des résultats de l'enquête de pratique religieuse de 1957, l'un des membres du clergé diocésain relève la «nécessité de l'industrialisation pour maintenir la population sur place»; il note sans déplaisir que «cette industrialisation s'est opérée jusqu'ici, chose exceptionnelle, sans provoquer une baisse importante de la pratique (dominicale)». Lors d'une conférence qu'il prononce alors devant des religieuses, le chanoine Boulard, établissant le même constat, en attribue le mérite à l'inspiration chrétienne du mouvement: «Je veux dire qu'au fond on a gagné la première manche, parce que l'industrialisation de cette région est assez ancienne, qu'elle s'est faite assez lentement, et que l'industrialisation qui, pratiquement, vient de Cholet, s'est faite sous l'inspiration de chrétiens qui ont orienté l'industrie pour donner à manger à tous ceux qui n'avaient pas à manger dans la région ...; ce sont des chrétiens qui ont pris la tête». Dans le rôle d'allié objectif du clergé, 


\section{Culture politique réfractaire et décollage économique}

le chef d'entreprise novateur, ainsi en charge d'une industrialisation qui ne saurait être moralement bonne que si elle est «diffuse» c'est-à-dire endogène et «paroissiale», tend de la sorte à se substituer progressivement au hobereau local. Il n'est évidemment pas sans intérêt de noter qu'il s'établit une «relation entre la vitalité paroissiale et la capacité d'entreprendre de chacun des bourgs» et que «les tentatives les plus nombreuses de création industrielle ... se sont manifestées dans les paroisses qui furent les plus animées durant une période suffisamment longue $» '$. Sans changer de cadre territorial, la capacité d'animation religieuse des élites locales s'est ainsi (ré)investie dans le champ économique.

Malgré l'affaiblissement sensible de sa position, ou en raison même de cet affaiblissement en ce qu'il est susceptible de faire place à d'autres formes de mobilisation et d'action, la paroisse continue de représenter un modèle à l'aune duquel s'évaluent les formes d'organisation locales nouvelles. Il a ainsi pu être observé «un lien relativement fort entre religion et dynamisme associatif $»^{2}$, non seulement parce que les fidèles de celle-là peuvent constituer les initiateurs et les cadres de celui-ci, mais aussi et surtout en raison de la fonction de «cohésion sociale» qui paraît s'attacher, notamment, à l'action culturelle. L'instance intégratrice change, mais demeure une logique qui privilégie et valorise l'intégration spatiale et la cohésion sociale, facteurs de consensus et d'identité locale. Le schème normatif ne s'est pas foncièrement transformé; le champ d'application du modèle matriciel s'est simplement déplacé. Le culturel s'est ici tendanciellement substitué au cultuel proprement dit.

\section{L'enseignement privé}

Cadre majeur de socialisation des nouvelles générations, l'école privée catholique constitue le support institutionnel déterminant d'une reproduction élargie du modèle culturel local. Elle tire son importance, dans le Bocage vendéen, de la position de quasi-monopole qu'elle s'y est acquise: au moment de l'enquête de sociographie religieuse de 1957, elle y scolarise en effet, selon les cantons, entre $89 \%$ des élèves du primaire (Pouzauges) et $99 \%$ (SaintFulgent), contre $30 \%$ dans le canton de Luçon, siège de l'évêché.

Par-delà le simple rapport de forces, en termes d'équipement scolaire et d'effectifs, entre l'enseignement public et l'enseignement privé, il convient de prêter attention à l'évolution interne et au processus d'innovation pédagogique de certains des établissements catholiques. Le collège Saint-Gabriel de Saint-Laurent-sur-Sèvre (canton de Mortagne-sur-Sèvre), créé en 1838, constitue à cet égard, on l'a vu, un exemple significatif non seulement d'adaptation mais bien d'innovation et d'anticipation par la création de sections d'enseignement technologique et, par là, la formation de la première relève des créateurs d'entreprises; l'évêque de Luçon le qualifie, au début du siècle, d'«école d'état-major de la Vendée militaire» ordonnée à la formation d'« officiers de l'armée catholique».

1. A. Chauvet, Porte nantaise et isolat choletais, op. cit., p. 181-182.

2. C. Vrignon, «Le développement culturel en milieu rural. L'exemple du département de la Vendée et de l'École de musique intercommunale Nord-Vendée», mémoire de second cycle, Institut d'études politiques de Bordeaux, 1994, p. 75. 


\section{Thierry Berthet, Jacques Palard}

Dès 1920, à l'instigation de l'Institut Catholique des Arts et Métiers de Lille (ICAM) et en application de la loi Astier du 25 juillet 1919 relative à l'organisation de l'enseignement technique, il se crée en son sein une École supérieure professionnelle dont l'objectif est de préparer aux carrières de l'industrie. Aux premières sections, axées sur les métiers du fer et du bois, viendront s'adjoindre les filières «chaussure » (1942), «électricité » (1948) et «électronique» (1958). Au cours des deux dernières décennies, se sont ouvertes successivement des sections de Brevet de technicien supérieur d'électronique et de Conception de produits industriels et, en 1993, une section Baccalauréat professionnel ${ }^{1}$. Il compte aujourd'hui plus de 2000 élèves, qui sont pour la moitié pensionnaires.

\section{La Jeunesse agricole catholique (JAC)}

Les mouvements catholiques de jeunes, apparus au cours de la seconde partie des années 1920, ainsi que les mouvements d'adultes représentent un important facteur de transformation tout à la fois du mode d'ancrage de nouveaux segments de l'Église catholique dans son environnement social et du fonctionnement interne de l'Église elle-même. Point n'est utile de revenir ici longuement sur ce qu'ont déjà abondamment montré de nombreuses études de sociologie rurale: le rôle modernisateur et mobilisateur de la JAC, dès avant la seconde guerre mondiale, tant sur le plan spécifiquement professionnel (équipement technique des exploitations, développement de nouvelles formes coopératives d'organisation du travail... $)^{2}$ que dans le domaine de «la prise en charge collective du milieu» et de l'engagement politique ${ }^{3}$.

Une dimension vaut sans doute ici d'être approfondie: les effets du recours, devenu systématique dans les mouvements d'Action catholique, à la

1. Cf. I. Merlet, «Le pensionnat Saint-Gabriel et ses élèves. Passé et présent: 1838-1987 », maîtrise d'histoire, Nantes, UER de Lettres, 1987. La conclusion de cette recherche, conduite par une étudiante devenue enseignante au sein de l'établissement, traduit parfaitement la posture singulière de l'observateur-participant dans un milieu social qui paraît imposer à ses membres une forme d'engagement et de forte implication et qui induit en l'occurrence une tendance "hagiographique»: «Le résultat de notre travail réside peut-être avant tout dans le fait d'avoir montré Saint-Gabriel comme une institution essentiellement régionale, née d'un besoin d'un pays qui a fait corps avec le sentiment exacerbé de l'honneur chrétien de ce pays de Vendée. Le pensionnat a su parfaitement s'adapter aux exigences de l'heure dans un véritable développement empirique.» (p. 137).

2. Cf. G. Gautreau, L'éclatement rural et les valeurs humaines, Paris, Editions Universitaires-UNMFREO, p. 122.

3. Selon J. Renard, c'est environ un tiers des communes qui, au milieu des années 1950 , sont touchées de façon décisive par la JAC: «Toute la frange orientale du Bocage vendéen est fortement marquée par le mouvement, notamment les communes du centre du Haut-Bocage» Géopolitique des Pays de Loire, op. cit., p. 325). L. Wylie souligne par ailleurs que, «dans une commune comme Chanzeaux, il n'y a véritablement que deux groupes de jeunesse qui comptent: la Jeunesse agricole catholique pour les jeunes gens; et la Jeunesse agricole catholique féminine, pour les jeunes filles ... Le type de jeunes qui y participent activement est assez clairement définissable : en général, il s'agit de quelqu'un du bourg, qui compte au moins un prêtre ou une religieuse parmi ses proches parents, qui professe des idées modernes aussi bien en ce qui concerne l'avenir du village que ses propres ambitions économiques et professionnelles. Enfin, c'est le plus souvent quelqu'un qui préférerait rester au pays et participer activement aux affaires de la paroisse plutôt que de s'expatrier», (Chanzeaux, village d'Anjou, op. cit., p. 281). 


\section{Culture politique réfractaire et décollage économique}

méthode de la «révision de vie»fondée sur le célèbre tryptique «VoirJuger-Agir». On a coutume d'en souligner le caractère pragmatique et empirique, qui fonde l'action non sur un savoir a priori mais sur une analyse de situations concrètes. Il convient également d'en relever les incidences sur les rapports entre les clercs et les laïcs ainsi qu'entre les fidèles pris individuellement et la communauté locale. Cette méthode délibérément inductive, qui fait appel à l'expérience personnelle et collective du militant et le situe non en lecteur passif du donné révélé ou dogmatique mais au contraire en position de «traducteur-interprétateur»', entraîne une transformation radicale, structurelle, des relations d'autorité entre acteurs religieux au sein de l'institution catholique. Parce qu'il occupe des zones d'incertitude qu'il est seul en mesure d'analyser et d'investir religieusement, le militant supplante d'un certain point de vue le clergé local, dont la capacité d'action est ainsi subordonnée à des formes de coopération avec «ses fidèles» totalement inédites et potentiellement conflictuelles. Dans un contexte où l'orthopraxie, comme fondement et base d'évaluation des comportements, peut entrer en concurrence avec l'orthodoxie, la «dépendance religieuse» caractéristique de la période antérieure s'amenuise sensiblement et, corrélativement, un processus d'individuation, déjà évoqué s'amorce et se développe, qui ne peut qu'être favorable à l'émergence d'un nouveau type de leadership au sein de la communauté locale. Sans doute insuffisant pour rendre compte à lui seul des nouvelles pratiques entrepreneuriales, ce processus d'individuation paraît toutefois avoir constitué une condition nécessaire de leur légitimité sociale et de leur efficacité économique.

Le développement de la JAC (puis du MRJC et des mouvements d'adultes) exerce également ses effets, au cours des années 1950-1960, au plan de la «lecture» des rapports sociaux: l'analyse sociale que pratiquent ses membres contribue à mettre en évidence la différenciation des «intérêts» culturels et socio-économiques des acteurs locaux. Appuyée par le jeune clergé, cette analyse introduit par là des ferments de rupture du consensus social, qui se traduisent notamment par la création des premières sections syndicales... ou par la critique du faste auquel donne lieu l'inauguration de tel nouvel équipement industriel ${ }^{2}$. Ce type d'effet des mouvements d'Action catholique ne touche cependant qu'une partie minoritaire de la population.

«Fragment» pétri d'un catholicisme traditionnel mais dynamisé au lendemain de la seconde guerre mondiale par un groupe d'entrepreneurs locaux qui ont fait ouvre de pionniers, le Nord-Vendée est le lieu de processus qui tirent leur originalité de leur complexité même. Tout semble se passer comme si la capacité d'innovation dont ont su faire preuve les nouveaux dirigeants industriels, tant sur le plan social que commercial ou proprement technique, était à la mesure de l'identité territoriale, laquelle s'est nourrie d'une longue et farouche opposition au système politique issu de la Révolu-

1. Cf. J. Palard, «D'un christianisme de position à un christianisme de mouvement(s)». Nouvelle revue théologique, 112 (6), novembre-décembre 1980.

2. Cf., par exemple, «Affrontement singulier au pays chouan: à propos de l'inauguration "fastueuse" d'un abattoir, le maire et le curé de Saint-Fulgent (Vendée) s'opposent en chaire devant les fidèles", Le Matin, 15-16 décembre 1979. 


\section{Thierry Berthet, Jacques Palard}

tion. En d'autres termes, la configuration d'ensemble s'est effectivement transformée, en particulier parce que de nouvelles élites économico-politiques, fondatrices d'un milieu industriel, se sont substituées aux précédentes, à dominante agraire. mais le changement est sans doute moins radical qu'il n'y paraît. Ainsi, l'ouverture sur l'extérieur, pour incontestable qu'elle soit. reste peut-être dictée, «en creux», par la traditionnelle méfiance à l'égard de l'Etat central, vis-à-vis duquel on s'autorise des pratiques de «contournement». C'est du moins l'une des interprétations qu'autorise l'analyse des résultats du référendum sur le traité de Maastricht: on peut légitimement estimer que l'adhésion à la thèse pro-européenne que traduit la confortable majorité remportée par le «oui» dans le fief même de Philippe de Villiers a quelque peu à voir avec la culture politique localement dominante. La «modernité » des allégeances et des comportements, inspirée par l'impératif de la continuité plus que par celui de la rupture, serait ainsi moins réelle qu'apparente. La notion d'actualisation conservatoire rendrait assez bien compte de la complexité des processus de changement et des rapports qui se nouent ainsi entre héritage et création. tradition et innovation.

Thierry Berthet est chargé de recherche CNRS au Centre d'étude et de recherche sur la vie locale de l'Institut d'études politiques de Bordeaux. Il est l'auteur de Seigneurs et colons de Nolvelle France. Cachan. Éditions de l'ENS-Cachan, 1992. Il a publié récemment (avec G. Bernier et D. Irwin) «Politiques et finances publiques: le cas du fédéralisme canadien», dans Annuaire des collectivités locales, 1996. Ses thèmes de recherche portent actuellement sur les politiques locales d'intégration des immigrants et sur les politiques de formation professionnelle (CERVL. Institut d'études politiques de Bordeaux, BP 101, 33405 Talence cedex).

Jacques Palard est directeur de recherche au CNRS, directeur du Centre d'études et de recherche sur la vie locale - Pouroir, Action publique, Territoire de l'Institut d'études politiques de Bordeaux. Il est l'auteur (en collaboration avec J. Lagroye, G. Lord, L. Mounier) de Les militants politiques dans trois partis français (PS, PC, UDR), Paris, Pedone, 1976 et de Pouvoir réligieux et espace social. Le diocèse de Bordeaux commé

1. L'animateur du Combat pour les valeurs, puis du Mouvement pour la France. citoyen des Herbiers (dont le canton a voté «oui » à $60 \%$ ), conseiller général du canton de Montaigu et président du Conseil général de la Vendée, s'est trouvé alors ouvertement contesté sur ses propres terres notamment par des représentants du milieu industriel, pour avoir confondu en quelque sorte conservatisme et réaction: cf. Le Mondt', 30 septembre 1996. L'envoyé spécial du journal observe que «beaucoup s'élèvent contre l'image déformée. encombrante et, pour tout dire, passéiste que Ph. de Villiers doune de la Vendée. C'est aussi cette irritation-là que les Vendéens du «oui" ont voulu faire percevoir. Leur pari sur l'Europe était le pari d'une Vendée ouverte. dynamique, moderne et sûre d'elle-même. Ils reprochent à $\mathrm{Ph}$. de Villiers de cultiver à l'envi "la mystique vendéenne"». 
organisation, Paris, Le Cerf, 1985. Il a publié récemment «Structural and Regional Planning Confronted with Decentralization and European Integration», Regional Politics and Policy, 3, 1994; «L'Église et le principe démocratique: synodalité et rapports de pouvoir", dans R. Luneau, P. Michel, Tous les chemins ne mènent plus à Rome, Paris, Albin Michel, 1995 et «Les effets institutionnels de la politique régionale de l'Union européenne», Politiques et Management public, 13 (3), 1995. Ses thèmes de recherche portent actuellement sur la transformation organisationnelle de l'Église catholique, sur les facteurs religieux d'intégration sociale en milieu urbain et sur la place de la région dans l'ingénierie institutionnelle, du niveau local au niveau international (CERVL, Institut d'études politiques de Bordeaux, Domaine universitaire, 33405 Talence cedex).

\section{RÉSUMÉ/ABSTRACT}

Les liens synallagmatiques qu'entretiennent, au sein d'une formation sociale, l'organisation du système politique et la composition du tissu économique permettent d'éclairer le processus du développement économique local. L'article souligne, dans un contexte d'industrialisation en milieu rural selon le modèle des districts industriels, les mécanismes de structuration de l'espace économique par les composantes socio-culturelles locales. La Vendée du Nord-Est constitue, à ce sujet, l'exemple intéressant d'un fragment où les synergies locales, de l'imprégnation des modes de faire-valoir agricoles traditionnels à la prégnance d'une culture religieuse tournée vers le renouvellement des élites locales, permettent l'émergence d'un système industriel marqué par l'endogénéité de son mode de développement et sa composition autour d'un réseau de petites et moyennes entreprises.

\section{REFRACTORY POLITICAL CULTURE AND ECONOMIC TAKEOFF IN NORTHEASTERN VENDÉE}

The bilateral links existing in a given social formation between the organization of the political system and the economic fabric shed light on the process of local economic development. The article emphasizes the structuring of economic space by local socio-cultural elements, in a context of industrialization in a rural setting, according to the model of industrial districts. Northeastern Vendée provides an interesting example of a fragment in which local synergies facilitate the emergence of an industrial system characterized by the endogeneity of its mode of development and the growth of a network of small - and medium - sized companies. These local synergies range from the penetration of traditional agricultural organization to the vitality of a religious culture oriented toward the renewal of local elites. 


\title{
CITOYENS INACHEVÉS \\ OU CITOYENS SUPÉRIEURS : EXEMPLES \\ ET QUESTIONS SUR L'INSTRUMENTATION DE LA CITOYENNETÉ RÉPUBLICAINE
}

\begin{abstract}
1) Une citoyenneté différée: cens civique et assimilation des indigènes dans les Établissements français de l'Inde 2) Une citoyenneté supérieure: l'improbable "fonction» des membres de la Légion d'honneur dans la République
\end{abstract}

L'une des questions les plus d'́licates aurquelles est confronté le' politiste est très certainement celle du statut de la notion de "citoyen", dont la Troisième République fit la base non seuleme'nt de son discours pédagogique mais, plus largement, de tout l'édifice démocratique républicain. Pour comprendre la nature d'une telle qualification, à la fois historiquement élolutive et aux registres multiples (juridique, social, constitutionnel, moral...), la nécessité s'impose désormais d'adjoindre à la saisic théoriqu' et idéelle la diversité concrèté de ses modalités d'établissement et de ses usages. Le renouvellement assez récent des approches do la citoyenn'té par les sciences humaines montre l'intér't't d'un travail réfl'xif et documenté sur l's expérienc's passées, en particulier dons la mesure où l'on se tient à distance d'une simple 't abusive idéalisation de l'œuvre républicaine. D'un "modèle républicain» dont le "citoy'n" est la pierre de touche, il faut aussi acc'pter et t'nter de repérer les fissures ét les failles.

Il convient, en somme, de' s'interroge'r. de manic'r' compléméntaire, sur les limites d'une appréhension de la coristruction sociale de la citoyenneté centrée sur l'affrontement de visions philosophiques ctlou religieuse's du monde' et de l'homme. La citojenneté est certes, en rípublique, le seul mode de distinction politique légitime. à la fois exclusif 't "universel»; toutefois cet "universalisme» exclusif. si souv'nt répété, a connu, au cour même du système politique, juridique et institutionnel républicain, d's aménagements singulièrement antinomiqu's avec l's valeurs fondatric'e's de la République.

Les deux article's qui suivent se proposent de présenter succinctement des terrains d'investigation favorables à cette entreprise: la mise en place dans certaines colonies françaises d'un réritable "'e'ns civique". dont la conséquence est la production de citoyens "partiels". "inachevés "; l'incorporation de l'ordre honorifique de la Légion d'honneur au sein des institutions républicaines, avec pour corollaire la difficile validation juridique et pseudo-fonctionnelle d'un type de "citoven décoré». «supérieur».

Damien Deschamps, Frédéric Caille 